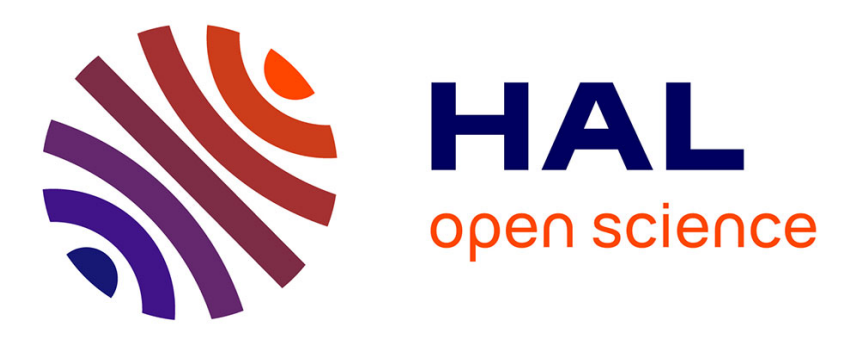

\title{
DSM-5, Trouble de personnalité schizotypique et nosographie psychanalytique structurale française
}

Cécile Prudent, Renaud Evrard, Nina Claude, Mélanie Laurent, Claude de Tychey

\section{- To cite this version:}

Cécile Prudent, Renaud Evrard, Nina Claude, Mélanie Laurent, Claude de Tychey. DSM-5, Trouble de personnalité schizotypique et nosographie psychanalytique structurale française. L'Évolution Psychiatrique, 2016, 81 (1), pp.176-190. 10.1016/j.evopsy.2015.03.002 . halshs-02137114

\section{HAL Id: halshs-02137114 \\ https://shs.hal.science/halshs-02137114}

Submitted on 22 May 2019

HAL is a multi-disciplinary open access archive for the deposit and dissemination of scientific research documents, whether they are published or not. The documents may come from teaching and research institutions in France or abroad, or from public or private research centers.
L'archive ouverte pluridisciplinaire HAL, est destinée au dépôt et à la diffusion de documents scientifiques de niveau recherche, publiés ou non, émanant des établissements d'enseignement et de recherche français ou étrangers, des laboratoires publics ou privés. 


\section{DSM-5, TROUBLE DE PERSONNALITE SCHIZOTYPIQUE ET NOSOGRAPHIE PSYCHANALYTIQUE STRUCTURALE FRANÇAISE}

Prudent, C., Evrard, R., Claude, N., Laurent, M., de Tychey, C. (2014). DSM-5, Trouble de personnalité schizoytpique et nosographie psychanalytique structurale française. L'Evolution psychiatrique.

\section{RÉSUMÉ}

Objectifs : Nous présentons dans cet article l'évolution des critères diagnostiques du DSM-5 pour le trouble de personnalité schizotypique qui est inclus à la fois dans les troubles de personnalité et dans le nouveau spectre schizophrénique. Les différentes interprétations (catégorielle versus pleinement dimensionnelle) de vécus assimilés à la psychose renvoient à des enjeux importants pour les approches contemporaines de la santé mentale. Un des nœuds du problème semble être le développement des notions paradoxales de schizotypie « saine », «bénigne » voire « joyeuse ». Nous réfléchissons sur l'option prise de privilégier l'approche dimensionnelle, en la mettant en parallèle avec la nosographie psychanalytique structurale française, en particulier avec la définition par Bergeret du caractère schizophrénique et les suppléances dans la clinique psychanalytique structurale lacanienne.

Méthode : Nous passons en revue les points communs entre ces modèles à partir d'une étude comparative de publications anglosaxonnes récentes dans le champ de la schizotypie et de travaux de clinique psychanalytique français.

Résultats : Les deux modèles jadis opposés se sont considérablement rapprochés avec l'acceptation dans le DSM 5 d'un point de vue dimensionnel, affirmant l'existence d'un continuum normal/pathologique en matière de fonctionnement et de traits de la personnalité. 
Discussion : Le DSM5 ne peut plus se prévaloir d'être athéorique. L'APA affirme d'ailleurs s'appuyer sur la théorie factorielle du Big Five pour définir domaine et traits de personnalité. mais l'APA omet de mentionner l'influence implicite des travaux de Kernberg.

Conclusion : Les auteurs proposent une approche plus intégrative, en considérant les mécanismes intrapsychiques sous-tendant le fonctionnement même de la personnalité et son expression symptomatique.

Mots clefs : DSM-5 - Trouble schizotypique - Nosographie psychanalytique structurale française - Approche intégrative - Continuum psychotique - Caractère schizophrénique psychotique

\title{
DSM-5, SCHIZOTYPAL PERSONALITY DISORDER, AND FRENCH STRUCTURAL PSYCHOANALYTIC NOSOGRAPHY
}

\begin{abstract}
Objectives: In this article we present the evolution in the diagnostic criteria of the DSM-5 regarding the Schizotypal personality disorder, which is included not only in the personality disorders but also in the new schizophrenia spectrum. The different interpretations (categorical versus fully dimensional) of experiences assimilated to psychosis refer to important issues in contemporary approaches to mental health. One of the knots of the problem appears to be the development of paradoxical notions of "healthy", "benign" or even "happy” schizotypy.
\end{abstract}

We examine the option taken to privilege the dimensional approach, placing it in parallel with the French structural psychoanalytical nosography, notably the definition by Bergeret of the schizophrenic character and the suppletions in Lacanian structural psychoanalytical clinical practice. 
Method: We have reviewed the common points between the new model of the DSM-5 and the French structural psychoanalytical model, using a comparative study of some main English language publications in the field of Schizotypy (Chmielewski \& al., 2014; Hori \& al., 2014; Rossell \& al., 2014; Nelson \& al., 2013; Ahmed \& al 2013; Tsuang \& al 2013; Van Os \& al., 2011, 2009; Lawrie \& al., 2010; Esterberg \& Compton, 2009; Nettle, 2006; First \& al., 2002; McCreery \& Claridge, 2002; Claridge, 1997; Siever \& al., 1991) [29, 31, 1, 2, 37, $52,14,15,18,38,28,3,26,22,13]$ and some French psychoanalytical works (Bergeret, 1974, 1986, 2012; Maleval, 2011; Trichet, 2011; Evrard, 2010, 2011, 2013a, 2013b; Chaperot, 2003; Fellahian, 2005) [6, 7, 8, 9, 10, 42, 16, 21, 43, 45, 46].

Results: The two formerly opposing models have drawn considerably closer with the acceptation of a dimensional perspective in the DSM-5. The choice of a dimensional rather than a categorical approach has undeniably contributed in reducing the interval between the psychiatric and French psychoanalytical nosography. Insofar as the new model of personality disorders - still waiting for empirical validation -focuses on the traits and functioning of the personality (assessed not only with regards to the self but also on interpersonal level, approached under the double aspect of the capacities of empathy and intimacy), it relies on far more stable aspects than the symptoms alone, and at the same time places them in a continuum between normal and pathological. We consider this as a real progress in the detection and understanding of the psychopathology.

Discussion: The DSM-5 can no longer prevail itself (as its authors have claimed for a long time) as being a-theoretical. Moreover, the American Psychiatric Association (APA) confirms that they rely on the Big Five factorial theory in defining the field of personality and its traits. However, the APA forgets to mention the implicit influence of the American psychoanalytical works of Kernberg, published earlier. Indeed the new scale of degrees of severity in the alteration of the functioning of the personality in the DSM-5, regarding the self and the 
interpersonal relationships, presents a fair number of similarities with the conceptualisations by Kernberg $(1995,1997,2004)$ [32-34] related to the degrees of alteration in self and in object relations, established by this author, so as to show the levels of severity in the different narcissistic pathologies. Nevertheless, we feel that the new options taken up by the APA are curiously contradictory. Although they are closer to the psychoanalytical model in the new conception proposed for personality disorders, they are in some respects surprisingly distant: the paranoia disorder has disappeared and only certain of its indicators are taken up (such as persecutory delusion) in a far too wide schizophrenia spectrum, and hence mistake the reality of the varying dissociation levels present in psychotic entities, already reported by psychiatric pioneers like Bleuler and by a great number of contemporary psychoanalytical psychopathologists. These levels are not rigorously organised in identical manner on the intrapsychic level and their clinical symptomatology during decompensation does not exactly take the same form. We suggest that substantively different levels of dissociation, leading to explicitly distinct symptomatologies, cover the globality of a spectrum that it would be more legitimate to called "psychosis" rather than "schizophrenia", whilst maintaining a distinction in the contemporary psychoanalytical model between schizophrenia, paranoid schizophrenia and paranoia.

Conclusion: The authors propose a more integrative approach. The latter would imply completing the level of symptomatic observation, of the dimensions of functioning and of the personality traits, by taking into consideration the intrapsychic mechanisms that underlie their expression, even if their objectivation would appear to all more difficult to formalise. The markers of these mechanisms refer notably to the nature of the conflicts experienced by the patient, to the nature of his/her distresses and dominating defences, as well as his/her object investment mode and his/her sites of conflictuality. 
Keywords: DSM-5 - Schizotypal personality disorder - French structural psychoanalytic nosography - Integrative approach - Psychotic continuum - Psychotic schizophrenic character 


\section{DSM-5, TROUBLE DE PERSONNALITE SCHIZOTYPIQUE ET NOSOGRAPHIE PSYCHANALYTIQUE STRUCTURALE FRANÇAISE}

\section{1) INTRODUCTION}

Longtemps négligé, le trouble de personnalité schizotypique (TPS) fait désormais l'objet de nombreux travaux au carrefour de la psychiatrie et de la psychologie (Pour deux revues récentes, voir : [1-2]). Depuis sa formulation originelle, sa place est située quelque part entre la «normalité » et la «schizophrénie ». Or, avec la montée en puissance des approches dimensionnelles et quasi-dimensionnelles dans la psychopathologie anglo-saxonne, le TPS connaît un regain d'intérêt en tant que syndrome intermédiaire potentiel au sein du spectre élargi de la schizophrénie [3].

Les objectifs de cet article sont pluriels :

- Présenter l'évolution des critères diagnostiques du DSM-5 [4] pour le TPS. Il est, à travers le nouveau manuel, défini de deux manières différentes: l'une classique, parmi les troubles de la personnalité, stricte duplication du DSM-IV TR [5]; et l'autre plus originale, en annexe, dans le nouveau modèle des troubles de la personnalité proposé par l'APA et nécessitant encore une validation par des recherches ultérieures. Cette présentation nous conduira à quelques réflexions critiques sur la catégorie des «troubles de la personnalité », visant à pointer les options discutables prises par l'American Psychiatric Association (APA), qui, dans son nouveau modèle, a fait disparaître deux troubles du groupe A du premier modèle : le trouble schizoïde et le trouble paranoïaque, tout en conservant le trouble schizotypique. Ce dernier est donc, de façon étonnante, inclus également dans le nouveau spectre schizophrénique et des autres troubles psychotiques, émargeant donc dans deux catégories diagnostiques différentes. Quels sont les enjeux de ces hésitations diagnostiques?

- Nous pencher sur l'option qui a été prise par l'APA dans le nouveau modèle, privilégiant une approche plus dimensionnelle que catégorielle des troubles. Nous souhaitons ici, 
montrer à travers l'exemple de la nouvelle conceptualisation du trouble TPS reclassé dans le spectre schizophrénique, que la nosographie psychiatrique du DSM-5 se rapproche considérablement d'une autre nosographie, présentée souvent comme opposée et inconciliable au modèle DSM, en l'occurrence la nosographie psychanalytique structurale française [6-10].

Pour élaborer cette réflexion, nous avons parcouru les sections du nouveau manuel du DSM-5. Nous présenterons d'abord au lecteur le double système de critères diagnostiques proposé pour le trouble de personnalité schizotypique, en retraçant brièvement son historique. Nous nous pencherons ensuite sur l'évolution des critères afférente au nouveau modèle et aux théories sous-jacentes. Dans un dernier temps, nous le mettrons en parallèle avec le modèle structural de la psychopathologie psychanalytique française, défendu par Jean Bergeret [6-8] et, sous une forme différente, par des psychanalystes lacaniens [9-10]. Nous détaillerons les points communs entre la description du DSM et celle proposée par Bergeret, pour rendre compte du caractère schizophrénique, mode d'expression normal compensé de la structure de personnalité schizophrénique. Au final, nous conclurons sur l'intérêt d'adopter une perspective intégrative en psychopathologie, au sens de Ionescu [11].

\section{2) ANALYSE DES CRITERES DU TROUBLE DE PERSONNALITE}

\section{SCHIZOTYPIQUE DANS LES DEUX MODELES PROPOSÉS PAR LE DSM-5}

\section{A. Le modèle classique copie du DSM IV-TR}

Il inclut le trouble schizotypique dans les groupe A des troubles de la personnalité, à coté du trouble paranoïaque et du trouble schizoïde de la personnalité, sans changer les critères qui le définissent. Le DSM-5 continue à poser qu'il constitue un modèle d'inconfort aigu dans les relations intimes, de distorsions cognitives et perceptives et d'excentricités du comportement. Le tableau 1 rappelle les critères diagnostiques du DSM-IV-TR : 
Tableau 1: Critères diagnostiques du trouble de personnalité schizotypique dans le DSM-IV-TR

\begin{tabular}{|c|c|}
\hline & $\begin{array}{l}\text { Mode général de déficit social et interpersonnel, marqué par une gêne aiguë } \\
\text { et des compétences réduites dans les relations proches, par des distorsions } \\
\text { cognitives et perceptuelles, et par des conduites excentriques. Le trouble } \\
\text { apparaît au début de l'âge adulte et est présent dans des contextes divers, } \\
\text { comme en témoignent au moins cinq des manifestations suivantes : }\end{array}$ \\
\hline 1 & idées de référence (à l'exception des idées délirantes de référence) \\
\hline 2 & $\begin{array}{l}\text { croyances bizarres ou pensée magique qui influencent le comportement et qui } \\
\text { ne sont pas en rapport avec les normes d'un sous-groupe culturel (par } \\
\text { exemple superstition, croyance dans un don de voyance, dans la télépathie ou } \\
\text { dans un « sixième sens »; chez les enfants et les adolescents, rêveries ou } \\
\text { préoccupations bizarres) }\end{array}$ \\
\hline 3 & perceptions inhabituelles, notamment illusions corporelles \\
\hline 4 & $\begin{array}{c}\text { pensée et langage bizarres (par exemple, propos vagues, circonstanciés, } \\
\text { métaphoriques, alambiqués ou stéréotypés) }\end{array}$ \\
\hline 5 & idéation méfiante ou persécutoire \\
\hline 6 & inadéquation ou pauvreté des affects \\
\hline 7 & comportement ou aspect bizarre, excentrique ou singulier \\
\hline 8 & $\begin{array}{c}\text { absence d'amis proches ou de confidents, en dehors des parents du premier } \\
\text { degré }\end{array}$ \\
\hline 9 & $\begin{array}{l}\text { anxiété excessive en situation sociale, qui ne diminue pas quand le sujet se } \\
\text { familiarise avec la situation, et qui est due à des craintes persécutoires plutôt } \\
\text { qu'à un jugement négatif sur soi-même }\end{array}$ \\
\hline
\end{tabular}




\section{B. Le nouveau modèle du trouble schizotypique dans le DSM-5.}

Les équipes de l'APA ont procédé ici à un choix surprenant. En effet, le trouble schizotypique est à la fois inclus dans le nouveau modèle des troubles de la personnalité à partir de critères diagnostiques bien différents, alors que disparaissent sans explication véritable de cette classification, le trouble schizoïde et le trouble paranoïaque. Par ailleurs, le trouble schizotypique apparait également dans le chapitre consacré au spectre schizophrénique et autres troubles psychotiques où il apparait sur un continuum comme la forme la moins grave d'un spectre qui va jusqu'au trouble schizo-affectif et à la catatonie.

En vérité, depuis ses origines, la notion de schizotypie possède un lien étrange à la pathologie en général et à la schizophrénie en particulier. Elle est fréquemment décrite comme un facteur de vulnérabilité individuelle à la schizophrénie, alors que cette évolution ne concernerait que $10 \%$ des sujets schizotypiques. La personnalité schizotypique, introduite dans le DSM-III, erre littéralement entre diverses catégories, accusant un manque de consensus suspect ([12], p. 279-280). Dans cette nomenclature américaine, la question de savoir si la personnalité schizotypique devait demeurer sur l'Axe II, l'axe des «troubles de la personnalité » (c'est-à-dire, dans la terminologie européenne, à la pathologie de la personnalité, aux caractères ou personnalités pathologiques) ou si elle ne serait pas mieux classée parmi les troubles de l'Axe I - par exemple en tant que variété clinique mineure de schizophrénie - avait été clairement posée lors de l'élaboration du DSM-IV [13]. Il fut décidé que la personnalité schizotypique n'impliquait pas une maladie mentale stricto sensu, du moins pour le DSM. Car, sur ce point précis, la classification internationale des maladies dans sa dixième révision (CIM-10) — a adopté en 1992 un point de vue différent de celui de l'APA en ne classant pas le trouble schizotypique parmi les troubles de la personnalité mais au sein de la rubrique F2 (comme les différentes formes cliniques de schizophrénie ou de psychoses délirantes considérées comme «fonctionnelles »). 
Alors que règne cette ambivalence sur le caractère pathologique ou non de la schizotypie, le rapprochement, s'il est effectué, sera toujours avec la schizophrénie. Dans le DSM-IV, les évolutions respectives de la personnalité schizotypique et de la schizophrénie sont considérées comme distinctes. Néanmoins, c'est le passage de l'une à l'autre qui est le plus souvent sousentendu, en dépit de l'ambiguïté des classifications et des résultats empiriques.

Le TPS s'inscrit dans un débat plus large sur les différentes interprétations du « continuum psychotique », concept qui s'appuie sur la prévalence élevée d'expériences assimilées à la psychose (hallucinations et délires, principalement) dans la population générale [14-16]. Les questions les plus controversées portent sur l'adéquation entre ces données épidémiologiques et psychométriques est les différents modèles - anciens comme nouveaux - visant à rendre compte de ce que serait, véritablement, la psychose. Les expressions de «phénotype psychotique » et de «spectre de la schizophrénie », employées à titre de métaphores de l'extension de ces modèles à une gamme enrichie d'expériences, se cristallisent dans l'entité «schizotypie» forgée par le psychanalyste Rado [17] à partir de la contraction de «schizophrenic phenotype ». Actuellement, il n'y a pas encore de consensus sur le modèle qui pourrait le plus efficacement rendre compte de l'ensemble des données (voir le débat dans le British Journal of Psychiatry suite à l'article de Lawrie et al. [18] ; voir plus récemment, en faveur du modèle pleinement dimensionnel: [2]). Certains pragmatiques affirment (par exemple [19]) qu'il faut conserver le modèle catégoriel car il est plus rapide à apprendre et plus facile à appliquer, en particulier dans les pays avec un faible PIB !

Un des champs de bataille se trouve être celui des croyances et expériences dites anomales, exceptionnelles, paranormales ou spirituelles [20-21]. Ces vécus, très répandus dans la population générale, sont interprétés comme des manifestations de ce phénotype psychotique. Or, depuis les années 1990, un ensemble d'étude a permis de dégager différents profils de schizotype [22], aboutissant à ce paradoxe : certaines personnes ont un score élevé dans les 
sous-échelles «positives» de la schizotypie (relatives aux croyances et perceptions inhabituelles) sans avoir ni un score élevé dans les sous-échelles «négatives », ni un profil psychopathologique avéré. Cela a pu être mis en évidence pour des personnes privilégiant une interprétation spirituelle ou paranormale de leurs expériences, sur un versant adaptatif bien que parfois via des modalités marginales (adhésion à des groupes ou croyances New Age, spirites, parapsychologiques, etc. : par exemple, [23-26]). D’où les notions éminemment paradoxales de « schizotypie saine », «joyeuse » ou «bénigne » [22, 26-27], qui formulent un des nœuds du problème.

\section{Influences des théories de la personnalité.}

La «dimensionnalisation » de la schizotypie s'est accentuée avec les travaux de chercheurs spécialistes de la personnalité et de ses troubles, à commencer par le psychologue Gordon Claridge [22], un disciple de Hans Eysenck. Leurs études ont amené à concevoir la schizotypie comme une dimension de la personnalité, une simple différence entre les individus, neutre sur le plan de la santé mentale (c'est-à-dire n'étant pas systématiquement associé à une morbidité psychopathologique). Ainsi, des scores élevés aux sous-échelles de désorganisation cognitive et d'expériences inhabituelles furent corrélés avec des aptitudes créatives et la réussite universitaire [28], l'ouverture à l'expérience [29] ou l'inclination à vivre et témoigner d'expériences anomales [30]. Dans l'étude de Hori et al. [31], les performances neurocognitives des personnes montrant à la fois des scores élevés de schizotypie positive et une bonne adaptation étaient comparables à celles des personnes adaptées avec des scores de schizotypie faibles, et supérieures à celles des personnes avec une schizotypie élevée mais mal adaptées. Ces recherches viennent suggérer une relation complexe et non-linéaire entre les traits schizotypiques et la psychopathologie.

Le nouveau modèle mis en place dans le DSM-5 a visiblement cherché à mieux intégrer ces travaux. Il a, selon nous, subi des influences à la fois implicites et explicites sur le plan 
théorique pour définir le fonctionnement de la personnalité. Avant de les présenter, nous rappellerons que dans son nouveau modèle, le DSM-5 se centre sur deux aspects cruciaux de la personnalité :

- le self (dans sa double dimension d'identité et d'auto-direction). Il est défini comme expérience de soi en tant qu'unique, avec des limites claires entre soi et les autres, avec une stabilité de l'estime de soi, une adéquation de l'évaluation de soi, la capacité et l'aptitude à réguler une variété d'expériences émotionnelles. L'auto-direction désigne, quant à elle, la poursuite de buts de vie cohérents et signifiants à court terme et l'aptitude à avoir une représentation de soi constructive.

- le niveau interpersonnel approché sous deux aspects :

- L'empathie définie comme la compréhension et la capacité d'apprécier les expériences et motivations des autres; la tolérance à des perspectives différentes, ainsi que la compréhension des effets de son propre comportement sur les autres.

- L’intimité évaluée par la profondeur et la durée du lien avec les autres, le désir et la capacité d'établir une relation étroite fondée sur la mutualité des échanges.

L'échelle du niveau de fonctionnement de la personnalité (Dimensional Assessment of Personality-Basis Questionnaire, DAPP-BQ) mise au point dans le DSM-5 utilise chacun de ces éléments pour différencier 5 niveaux d'altérations de la personnalité, allant de faible ou pas d'altération (niveau 0 : fonctionnement adaptatif, sain, à un peu d'altération (niveau 1), puis altération modéré (niveau 2) à sévère (niveau 3) et extrême (niveau 4).). L'altération dans le fonctionnement de la personnalité et les traits de personnalité pathologiques est relativement stable dans une variété de contextes personnels et sociaux. Le terme relativement reflète le fait que toutes les personnalités, à l'exception des plus pathologiques, montrent quelques degrés d'adaptabilité. Cette échelle repose donc sur une conception dimensionnelle et non plus catégorielle de la personnalité, avec l'idée d'un continuum entre le 
fonctionnement normal et le fonctionnement pathologique, qui rejoint la conception de la nosographie psychanalytique structurale française.

Mais nous pensons que cette nouvelle conceptualisation des niveaux de gravité de l'altération du self et du fonctionnement interpersonnel a subi implicitement une autre source d'influence psychanalytique structurale aux USA, celle représentée par le psychiatre psychanalyste américain Otto Kernberg [32-34] et les psychiatres américains formés par lui dont certains font sans doute encore partie du staff de l'APA actuel. Kernberg est une figure importante de la psychanalyse internationale puisqu'il a été président de l'Association de Psychanalyse Internationale (API). Dans un entretien accordé à Benvenuto et Siniscalco, réalisé en 1996 [35], Kernberg qui a collaboré directement aux premières versions du DSM est interrogé sur l'évolution du DSM-IV par rapport à la nosographie psychanalytique. Il soulignait à l'époque que le domaine le plus pauvre dans le DSM III et IV était celui relatif aux troubles de la personnalité et aux névroses symptomatiques. Il est en même temps très critique vis-à-vis du DSM, remarquant avec beaucoup d'humour que « le DSM veut classer sur un mode descriptif les syndromes majeurs d'une manière a-théorique, quoique ce qui se dit a-théorique reflète bien souvent une théorie non reconnue sous-tendant un empirisme assez simpliste et un préjugé non reconnu contre la psychanalyse. » Il avait selon nous une juste vision de l'avenir lorsqu'il concluait son entretien en disant: «Des efforts sont actuellement menés pour modifier et enrichir le DSM IV avec l'incorporation des découvertes psychodynamiques, et nous verrons des changements significatifs dans notre système de classification avec le temps que cela prendra en arrivant au DSM V et VI. ». En effet la nouvelle échelle des degrés de gravité de l'altération du fonctionnement de la personnalité, au niveau du self et des relations interpersonnelles, présente un certain nombre de ressemblances avec les conceptualisations élaborées dans une perspective psychanalytique par Kernberg il y a une quinzaine d'années qui, à notre avis, leur a servi d'inspiration. Nous faisons ici référence précisément aux degrés 
d'altérations du self et de la relation d'objet, établis par cet auteur pour rendre compte des niveaux de gravité des différentes pathologies narcissiques, pointées déjà à cette époque comme en augmentation exponentielle et relevant de prises en charge spécifiques...

La dernière source d'influence - cette fois explicitement reconnue - de cette nouvelle conceptualisation du DSM-5 est manifestement la théorie factorielle du Big Five, car c'est elle qui a servi de base aux psychiatres de l'APA pour définir les domaines et traits de personnalité. En effet, les traits pathologiques de personnalité sont organisés en 5 domaines larges : affectivité négative, détachement, antagonisme, désinhibition et psychoticisme. À l'intérieur de ces 5 domaines de traits larges, il y a 16 facettes de traits spécifiques qui ont été développés à partir d'une revue des modèles de traits existant, et de manière subséquente, à travers des recherches répétées sur des échantillons de personnes recherchant des services de santé mentale [36].

L'altération du fonctionnement de la personnalité prédit la présence d'un trouble de la personnalité. La sévérité de l'altération prédit si l'individu a plus d'un trouble de la personnalité, ou un seul trouble typique sévère de la personnalité. Un niveau modéré d'altération du fonctionnement de la personnalité est exigé pour poser le diagnostic de trouble de la personnalité.

Les nouveaux critères diagnostiques du trouble de la personnalité schizotypique sont alors les suivants (Tableau 2) :

Tableau 2 : Nouveaux critères diagnostiques du trouble de la personnalité schizotypique dans le DSM-5

A. Altération modérée ou plus grande du fonctionnement de la personnalité, manifesté par des difficultés dans deux des 4 sphères suivantes : 


\begin{tabular}{|c|c|}
\hline 1 & $\begin{array}{l}\text { Identité : limites confuses entre soi et les autres, concept de soi distordu, } \\
\text { expression émotionnelle souvent non congruente avec le contexte ou } \\
\text { l'expérience interne. }\end{array}$ \\
\hline 2 & $\begin{array}{l}\text { Auto-direction : buts irréalistes ou incohérents, pas d'ensemble clair des } \\
\qquad \text { standards internes. }\end{array}$ \\
\hline 3 & $\begin{array}{l}\text { Empathie : difficulté prononcée à comprendre l'impact de ses propres } \\
\text { comportements sur les autres, fréquentes mauvaises interprétations des } \\
\text { motivations et comportements des autres. }\end{array}$ \\
\hline 4 & $\begin{array}{l}\text { Intimité : altération marquée du développement des relations étroites, associée à } \\
\qquad \text { la méfiance et à l'anxiété. }\end{array}$ \\
\hline \multicolumn{2}{|r|}{ B. Présence de 4 ou plus des six traits de personnalité pathologique suivants : } \\
\hline 1 & $\begin{array}{l}\text { Dérégulation cognitive et perceptive (un aspect du psychoticisme): processus } \\
\text { de pensée inhabituels, bizarres ou vagues, fondés sur des présomptions, } \\
\text { métaphoriques, surélaborés; ou pensée et parole stéréotypées, sensations } \\
\text { bizarres à partir de modalités sensorielles variées. }\end{array}$ \\
\hline 2 & $\begin{array}{l}\text { Expériences et croyances inhabituelles (un aspect du psychoticisme) : les } \\
\text { contenus de pensée et les visions de la réalité, lorsqu'ils sont vues par les autres, } \\
\text { apparaissent bizarres et idiosyncrasiques, correspondant à des expériences } \\
\text { inhabituelles de la réalité. }\end{array}$ \\
\hline 3 & $\begin{array}{l}\text { Excentricité (un aspect du psychoticisme): comportement ou apparence } \\
\text { curieux, bizarres ou inhabituels, tendance à dire des choses inhabituelles ou } \\
\text { inappropriées. }\end{array}$ \\
\hline 4 & $\begin{array}{l}\text { Affectivité restreinte (un aspect du détachement): réaction faible à des } \\
\text { situations déclenchant des émotions, expériences et expression émotionnelles }\end{array}$ \\
\hline
\end{tabular}




\begin{tabular}{|l|l|}
\hline & restreintes , indifférence ou froideur. \\
\hline 5 & Retrait (un aspect du détachement) : préférence à être seul plutôt qu'avec les \\
autres ; réticence dans les situations sociales, évitement des contacts et des \\
activités de nature sociale, manque d'initiation au contact social.
\end{tabular}

Ces critères étant posés, nous souhaitons pointer le fait que dans ce modèle, rappelons-le dimensionnel, le DMS-5 reconnait que l'individu peut présenter une altération légère du fonctionnement de la personnalité non synonyme de pathologie. Si cette altération est de ce niveau, elle prendra sur le plan clinique les formes suivantes sur le plan du fonctionnement de sa personnalité :

- $\quad$ Au niveau de l'identité : le sujet aura alors pour le DSM-5 un sens relativement intact de son self, avec quelques affaiblissements de le repérage de ses limites lors de la confrontation à des émotions fortes, ou lors d'une détresse mentale. Il aura aussi une estime et une perception de lui-même un peu distordues, avec des émotions fortes pouvant être source de détresse associées à une restriction du vécu émotionnel.

- $\quad$ Au niveau de l'auto-direction : la personne située à ce niveau d'altération pourra avoir une variété de standards personnels irréalistes ou socialement inappropriés limitant quelques aspects de son épanouissement. Elle sera cependant capable de réfléchir sur son expérience intérieure, mais pourra exagérer la singularité d'un type de connaissance de soi (intellectuelle, émotionnelle).

- $\mathrm{Au}$ niveau interpersonnel: les conséquences sur l'empathie et l'intimité seront également relativement réduites : 
- Sur le plan de l'empathie : celle-ci sera certes un peu compromise au niveau de son aptitude à apprécier et comprendre les expériences des autres, ce qui pourra le pousser à voir les autres comme ayant des attentes irraisonnables, ou susciter un désir de les contrôler. Mais il subsistera une capacité à considérer et à comprendre différentes perspectives malgré une tendance à résister à le faire, avec une conscience variable de l'effet de son propre comportement sur les autres.

- Sur le plan des relations intimes : le sujet fonctionnant à ce niveau restera capable d'établir des relations durables dans sa vie personnelle et communautaire, avec quelques limitations dans leur degré de profondeur et de satisfaction. Il pourra être capable de former ou de désirer former des relations intimes et réciproques, mais pourra être inhibé et gêné si des émotions intenses ou des conflits surgissent. Le DSM5 nous précise également que la coopération pourra dans ce cas être inhibée par des standards un peu irréalistes, limitant l'aptitude du sujet à respecter ou répondre aux comportements, idées ou émotions des autres.

\section{3) PARALLELE ENTRE LE NOUVEAU MODELE DSM-5 DU TROUBLE} SCHIZOTYPIQUE ET LE MODELE PSYCHANALYTIQUE STRUCTURAL

Le débat sur la schizotypie du début des années 1990 influença plus généralement la théorisation d'autres entités cliniques, jusqu'à favoriser la transition du DSM d'un modèle catégoriel (trouble morbide présent ou non), à un modèle quasi-dimensionnel (continuum allant de formes bénignes à des formes sévères, la schizophrénie en étant une extrémité). Mais, pour de nombreux observateurs, le DSM-IV et sa version révisée n'étaient toujours pas conformes aux travaux qui semblaient montrer la supériorité d'un modèle pleinement dimensionnel où l'axe psychopathologique croise un axe de personnalité qui vient moduler son expression [37-38, 22]. 
C'est que la généralisation d'une telle approche pleinement dimensionnelle vient poser un problème épistémologique fondamental à la nosographie américaine fondée sur un découpage séparant des troubles majeurs cliniques (Axe I) et des troubles de la personnalité (Axe II). En effet, cette approche - dite également approche hybride ou composite - incorpore des éléments issus du modèle médicale et du modèle psychologique. Le découpage institué en axes distincts en devient caduc à partir du moment où il est reconnu que la construction subjective de la personnalité médiatise la forme prise par la schizotypie : soit sous la forme d'un trouble clinique ; soit sous la forme plus diffuse d'un trouble de la personnalité ; soit sous une forme «saine », que l'on pourrait dire « compensée », « supplée » ou «élaborée », qui échappe de fait à une entreprise purement nosographique.

La part des traumatismes dans l'enfance (par exemple, abus sexuels ou maltraitances physiques) et des événements de vie négatifs dans l'émergence, à l'adolescence et dans l'ensemble de la vie, d'expériences et de symptômes assimilés à la psychose [39-41] vint également relancer l'intérêt pour des approches psychodynamiques, issues de la clinique janétienne ou freudienne, esquissant un rapprochement entre la schizotypie et certaines formes de névroses [42] ou avec d'hypothétiques «états extraordinaires » des structures névrotiques ou psychotiques [43].

Nous avons souhaité donner au lecteur ce descriptif détaillé pour le mettre en parallèle avec celui de la nosographie psychanalytique structurale française, à travers deux courants : celui défendu par Jean Bergeret [6-8] depuis de nombreuses années ; et la clinique structurale lacanienne.

\section{A. Le caractère schizophrénique selon Bergeret}

Bergeret affirme en effet depuis fort longtemps la nécessité d'une approche dimensionnelle et soutient, dans son approche structurale, l'idée que tout mode d'organisation 
de la personnalité, qu'il soit névrotique, limite narcissique ou psychotique (c'est nous qui soulignons) peut fonctionner sur un mode normal compensé, c'est-à-dire adapté à la fois à sa réalité interne et à la réalité externe. Il peut aussi s'exprimer sur un mode instable (porteur alors d'expressions symptomatiques), ou carrément décompensé (traduisant alors une double rupture interne et externe) lors de la rencontre de conflits internes et (ou) externes non élaborables, dépassant les ressources adaptatives et défensives du sujet. Le nouveau modèle dimensionnel des troubles de la personnalité et de la psychopathologie du DSM-5 nous semble incontestablement se rapprocher de ce point de vue. Il suffit pour s'en rendre compte de mettre en parallèle le descriptif clinique du trouble schizotypique de la personnalité, à son niveau d'altération le plus faible, avec le descriptif clinique sémiologique du caractère schizophrénique, mode d'expression normal compensé de la structure schizophrénique formalisé par Bergeret [6]. Ainsi l'APA dans le DSM-5 [4] précise-t-elle que :

«les caractéristiques typiques du trouble de la personnalité schizotypique sont des altérations dans la capacité d'établir des relations sociales et des relations étroites, et des excentricités sur le plan cognitif. Les altérations sur le plan perceptif et du comportement, sont associées à une image de soi distordue, à des buts personnels incohérents et accompagnées d'une expression émotionnelle réduite et de méfiance »

Mais nous ajouterons, de notre côté, qu'en reprenant les critères proposés, le niveau d'altération du fonctionnement de la personnalité doit être au moins modéré pour parler de trouble de la personnalité schizotypique. Toutefois, ce qualificatif cesse d'être apposé sur le plan diagnostique, lorsque le niveau d'altération du fonctionnement de la personnalité est léger ou absent. Ne serait-il pas dès lors légitime de parler d'un TPS cliniquement normal?

Le parallèle avec le caractère schizophrénique, mode d'expression normal de la structure schizophrénique de personnalité défendue par Bergeret s'impose alors. Cet auteur 
souligne que dans cette configuration, le sujet va présenter des traits de comportements marqués «par le maniérisme, la tendance à l'isolement, à la rêverie, à la bizarrerie, à la jalousie ou bien à l'insécurité » ([6], p. 209). Bergeret ajoute ensuite que :

« le caractère schizophrénique se reconnait extérieurement à sa carence dans le contact, sa tendance au repliement sur soi-même, à une activité intérieure assez intense ; on peut cependant voir apparaître dans ce fonctionnement quelques petits éléments illogiques. Le peu de chaleur affective qui se dégage du contact, la froideur du comportement relationnel, le désintérêt objectal souvent assez évident, spécifient l'abord de tels sujets. »

Nous pouvons également faire valoir une autre convergence dans le choix de privilégier un nouveau modèle de la personnalité, à la fois plus intégratif et plus dimensionnel que catégoriel, qui rejoint deux autres aspects importants de la position bergerétienne. D'abord, celle d'un continuum normal/pathologie pour toute personnalité : l'APA le reconnait implicitement lorsqu'elle déclare dans le nouveau manuel que «toutes les personnalités, excepté les personnalités pathologiques les plus sévères, présentent quelques degrés d'adaptabilité » ([4], p. 763). En corollaire, dans la section du manuel réservée aux nouveaux outils d'évaluation, cette perspective pleinement dimensionnelle en termes de continuum normal-pathologique est patente, tant dans l'échelle d'évaluation des symptômes (allant d'un gradient d'absent à sévère), que dans les échelles d'évaluation des dysfonctionnement du self et des relations interpersonnelles.

Ensuite, nous pensons qu'un progrès important a été réalisé dans le DSM-5 en privilégiant les dimensions du fonctionnement et les traits de la personnalité aux seuls symptômes. L'APA note en effet que : 
«Les altérations dans le fonctionnement et les traits de personnalité sont relativement stables. Les traits de personnalité, les dispositions à se comporter, ou à sentir les choses d'une certaine façon, sont plus stables que les expressions symptomatiques de ces dispositions. » ([4], p. 763)

Cette position est très proche de la perspective structurale défendue par Bergeret [6-7] dans la nosographie psychanalytique française qui affirme l'existence de constantes à la fois dans l'expression comportementale et dans les mécanismes intrapsychiques reliés au fonctionnement de la personnalité qui le sous-tendent.

Dans ces conditions, on ne sera pas surpris de notre rapprochement entre les descriptifs cliniques donnés par l'APA du trouble de la personnalité schizotypique à l'intérieur du spectre schizophrénique, où elle représente le niveau potentiel de pathologie psychotique le moins sévère, et le caractère schizophrénique repéré par Bergeret [6].

\section{B. Les suppléances dans la clinique structurale lacanienne}

Dans la perspective d'une clinique différentielle d'orientation lacanienne, Arce-Ross propose de concevoir une distinction triadique entre structure, forme clinique et suppléance, qu'il applique ainsi à la psychose :

$«(\ldots)$ une structure psychotique peut se maintenir longtemps, parfois toujours, plus ou moins stabilisée, c'est-à-dire sans conséquences cliniques ; la structure psychotique, en se décompensant à partir de la rencontre avec un élément réel, peut se transformer en psychose clinique; mais, grâce à un nœud symptomatique créé spécialement par un rebiffement du sujet, la psychose clinique peut trouver une suppléance qui la fait en partie revenir à l'état initial (sans manifestations cliniques) et en partie accéder à un état nouveau. » ([44], p. 409 ; ses italiques) 
Le repérage d'une structure aurait ainsi un intérêt clinique, que cette structure se présente avec ou sans manifestations symptomatiques, et avec ou sans suppléances apparentes; donc en parallèle d'un continuum normal-pathologique. L'approche structurale lacanienne offre également la possibilité d'appréhender les expériences réputées psychotiques dans toute leur complexité, en leur trouvant une juste place en s'émancipant de la polarité normalepathologique. Une hypothèse possible serait de prendre en considération des oscillations entre des états ordinaires et des états extraordinaires des structures psychiques [43].

Ce discernement devrait porter sur un moment de la structure, sans figer le diagnostic par des catégories ayant des effets iatrogéniques de stigmatisation et de chronicisation [45]. Il faudrait donc distinguer la logique de la structure avant le déclenchement de la psychose, pendant le déclenchement et la structuration faisant suite au déclenchement. De même, dans les modes de suppléance employés par le sujet pour parer à la psychose, il faudrait distinguer celles qui ont une fonction préventive de celles qui ont une fonction curative. De sorte que la même idée inhabituelle voire délirante n'aura pas la même valeur clinique selon la manière dont elle s'inscrit dans une logique structurale singulière [10].

Le déséquilibre initial de l'approche freudienne en faveur du modèle de la névrose perçue comme plus propice à une vie mentale adaptée - fut véritablement renversé par les théorisations de Lacan à la fin de son parcours. Comme les résume Fellahian ([46], p. 58), « la psychose, "lapsus du nœud", n'est plus l'inéluctable conséquence de l'échec du refoulement originaire et le symptôme, dans sa fonction de suppléance, devient un instrument d'équilibration de la structure. » La structure psychotique est ainsi mise en demeure de « serrer son nœud» au moyen d'un symptôme ou d'un sinthome, associées parfois à des créations artistiques ou à des découvertes scientifiques. Le cas de l'écrivain irlandais James Joyce est paradigmatique [47]. Et ce principe sera étendu pour chacune des structures dans la « théorie généralisée du symptôme ». 
Tous les pare-psychoses ne possèdent probablement pas la même efficacité stabilisante, et les travaux sur le «néo-déclenchement » appellent d'autres travaux sur l'efficacité variable des compensations [10]. Par ce biais, la clinique structurale peut tirer profit des recherches sur les stratégies d'ajustement (de coping) mises en place plus ou moins spontanément par les personnes vivant des expériences réputées psychotiques [48-49]. La psychopathologie est ainsi toujours mise en perspective avec les défenses subjectives qui permettent à certains sujets de compenser le déterminisme objectif de leurs troubles. Eugen Bleuler avait déjà observé la variabilité de la dissociation dans la schizophrénie, celle-ci pouvant parfois être latente ou à peine décelable. Cette dimensionnalité serait-elle la manifestation d'une efficacité plus ou moins grande des mécanismes supplétifs dans la psychose, dans une acception plurielle du « Nom du Père »?

Là où certains voient des différences de degré faisant de la psychose une maladie plus grave que la névrose, principalement du fait de la «perte de la réalité » (hallucinations et délires), selon la psychopathologie psychanalytique structurale, il s'agit de différences de nature, c'està-dire de modes d'organisation différents. Les données du «continuum psychotique » montrent que les personnes ayant une structure psychotique sont potentiellement plus nombreuses qu'on ne le pense généralement, ce qui nous invite à mieux examiner comment ces personnes font pour que leur psychose ne se déclenche pas ou plus. A l'image du fou incapable de s'intégrer dans la société, et même dangereux pour elle comme le suggèrent les médias, il faut substituer la conception d'une structure psychotique capable de s'adapter socialement, de devenir «ordinaire» au prix d'un certain effort, qui n'implique pas nécessairement l'abrasion du délire et des hallucinations [9].

$\mathrm{Au}$ final, il nous semble que les descriptions cliniques faites par les partisans des deux orientations psychiatriques et structurales sont empreintes de la même finesse et convergentes au moins sur certains points. En définitive, il nous apparait que le nouveau modèle du DSM-5 
et la nosographie psychanalytique structurale, que les cliniciens ont souvent opposés dans le passé en fonction de leur propre formation, tendent à se rapprocher...

\section{4) POUR CONCLURE}

L'hésitation diagnostique se faisait ressentir au temps du DSM-IV - adepte d'une approche quasi-dimensionnelle où la schizotypie apparaissait parmi les troubles de la personnalité - et de la CIM-10, plus catégorielle, dans laquelle la schizotypie figurait parmi les troubles majeurs du spectre de la schizophrénie. La mutation opérée à l'occasion de la parution du DSM-5 montre une volonté d'intégrer certaines avancées empiriques et théoriques en proposant à la fois de conserver le modèle quasi-dimensionnel, tout en s'ouvrant, dans une partie consacrée aux entités cliniques nécessitant davantage de recherches, à une approche pleinement dimensionnelle.

Nous trouvons cependant les nouvelles options prises par l'APA singulièrement contradictoires. Si elles se rapprochent du modèle psychanalytique dans la nouvelle conceptualisation proposée pour les troubles de la personnalité, elles s'en éloignent de manière surprenante en faisant disparaître complètement le trouble paranoïaque pour n'en reprendre que certains indicateurs (comme le délire de persécution) dans la classification du trop large spectre schizophrénique, méconnaissant du coup la réalité de niveaux de dissociation différents présentés par les entités psychotiques, comme le pointaient déjà les pionniers de la psychiatrie comme Bleuler et comme l'affirment un grand nombre de psychopathologues psychanalystes contemporains. Ces niveaux ne sont pas rigoureusement organisés de manière identique sur le plan intrapsychique et leur symptomatologie sur le plan clinique lors d'une décompensation ne prend pas rigoureusement la même forme. Nous posons que des niveaux de dissociation foncièrement différents, donnant lieu à des symptomatologies spécifiques différentes, recouvrent l'ensemble d'un spectre qu'il serait plus 
légitime d'appeler psychotique que schizophrénique, avec une distinction maintenue dans le modèle psychanalytique contemporain entre schizophrénie, psychose schizoparanoïde et paranoïa.

Avant même sa traduction française à venir, le nouveau manuel DSM-5 a déjà suscité des discussions et interrogations assez vives à l'intérieur de la psychiatrie française [50-51]. Une partie de ces discussions ont porté sur cet élargissement du phénotype psychotique à un ensemble d'expériences réputées psychotiques, sans être associées à tous les critères permettant de porter un diagnostic de psychose. En particulier, l'équipe du DSM-5 discuta la proposition d'une nouvelle entité : le Syndrome de risque de psychose, devenue Syndrome de psychose atténuée, écartée de justesse pour ne figurer finalement que dans la section 3 des annexes de cette dernière édition, invitant à davantage de recherches [52-54]. Les enjeux commerciaux semblaient prendre le pas sur l'intérêt scientifique et clinique, avec cette approche ciblant les adolescents et les jeunes adultes en proie à des épisodes d'hallucinations et de délires (généralement transitoires) et traités comme des psychotiques en puissance [55].

Le DSM-5 n'a pas encore opté clairement pour une approche pleinement dimensionnelle accueillant cette catégorie de «psychose bénigne » ou «saine », ce qui est tout à fait compréhensible pour un traité nosographique fondé sur des postulats objectivistes, se leurrant sur le fonctionnement de la réalité psychique [56]. A quand un DSM-6 où l'hallucination et le délire seront entendus comme des expressions subjectives d'une souffrance ne présageant nullement d'une entrée dans une forme de morbidité chronique?

Pour le clinicien d'orientation psychodynamique, la possibilité de privilégier une approche pleinement dimensionnelle par rapport à une approche catégorielle ou quasidimensionnelle a incontestablement contribué à réduire l'écart entre la nosographie psychiatrique et la nosographie psychanalytique structurale. Le nouveau modèle à valider des 
troubles de personnalité, en se centrant davantage sur le fonctionnement de la personnalité et les traits de personnalité, autrement dit sur des aspects beaucoup plus stables que les seuls symptômes, qui plus est dans une perspective de continuum entre le normal et le pathologique, nous apparait comme un vrai progrès dans le repérage et la compréhension de la psychopathologie. Il va assurément contribuer à réduire le problème important constitué par les co-morbidités et les troubles non spécifiés, sans pour autant le supprimer complètement. Pour y parvenir, nous faisons la proposition d'aller encore plus loin et de privilégier une approche pleinement intégrative. Cette dernière impliquerait de compléter le niveau de l'observation symptomatique, des dimensions du fonctionnement et des traits de la personnalité, par une prise en considération des mécanismes intrapsychiques qui sous-tendent leur expression, même si leur objectivation paraîtra à tous plus difficile à formaliser. Les marqueurs de ces mécanismes, qui sont autant de constantes venant organiser chaque personnalité, ont été clairement formalisés dans le modèle de la nosographie psychanalytique structurale française. Ils renvoient notamment à la nature des conflits vécus par le sujet, à la nature de ses angoisses et mécanismes de défenses dominants, ainsi qu'à son mode d'investissement des objets et aux lieux de la conflictualité. Nous suggérons aux recherches futures de les prendre en considération, en s'efforçant de les opérationnaliser, afin de mettre à l'épreuve leur capacité à affiner le diagnostic différentiel en clinique, à partir des nouveaux points de repère déjà disponibles.

Conflit d'intérêt : aucun. 


\section{Références}

1. Rosell DR, Futtermann SE, McMaster A, Siever LJ. Schizotypal personality disorder: a current review. Current Psychiatry Reports $2014 ; 16(7): 452$.

2. Nelson MT, Seal ML, Pantelis C, Philipps LJ. Evidence of a dimensional relationship between schizotypy and schizophrenia: a systematic review. Neuroscientific Biobehavioral Rev 2013 ; 37(3) : 317-27.

3. First MB, Bell CC, Cuthbert B, Krystal JH, Malison R, Offord DR, Riess D, Shea T, Widiger T, Wisner KL. Personality Disorders and Relational Disorders. In : Kupfer DJ, First MB, Regier DA, A Research Agenda for DSM-V. American Psychiatric Association, Washington DC, USA ; 2002 ; 125-152.

4. American Psychiatric Association. Diagnostic and Statistical Manual of Mental Disorders DSM-5.American Psychiatric Association, Washington DC, USA ; 2013.

5. American Psychiatric Association. Diagnostic and Statistical Manual of Mental Disorders DSM-IV-TR. American Psychiatric Association, Washington DC, USA ; 2000.

6. Bergeret J. La personnalité normale et pathologique. Paris : Dunod ; 1974.

7. Bergeret J. Abrégé de Psychologie pathologique. Paris : Masson ; 1986.

8. Bergeret J. Conversations libres. Psychomédia $2012 ; 33: 40-49$.

9. Maleval JC. Logique du délire (3 éd). Rennes : Presses Universitaires de Rennes ; 2011.

10. Trichet Y. L'entrée dans la psychose. Approches psychopathologiques, clinique et (auto-)traitements. Paris : Presses Universitaires de Rennes ; 2011.

11. Ionescu S. Quatorze approches de la psychopathologie ( $3^{\mathrm{e}}$ éd.). Paris : Armand Colin ; 2005.

12. Garrabé J. La schizophrénie : un siècle pour comprendre. Paris : Les Empêcheurs de Penser en Rond ; 2003.

13. Siever LJ, Silverman JM, Bernstein D. Schizotypal personality disorder: A review of its current status. J Pers Disorders $1991 ; 5$ : 178-93.

14. Van Os J, Hanssen M, Bijl RV, Vollenbergh W. Prevalence of psychotic disorder and community level of psychotic symptoms: an urban-rural comparison. Arch Gen Psychiatry $2011 ; 58: 663-8$.

15. Van Os J, Linscott R, Myin-Germeys I, Delespaul P, Krabbendam L. A Systematic Review and Meta-Analysis of the Psychosis Continuum: Evidence for a Psychosis Pronenes - persistence - impairment Model of Psychotic Disorder. Psychol Med $2009 ; 39(02): 179-95$.

16. Evrard R. Les expériences réputées psychotiques dans la population générale : essai de problématisation. Ann médico-psychologiques 2011 ; 169(5) : 282-7.

17. Rado S. Dynamics and classification of disordered behaviour. Am J Psychiatry 1953 ; 110 : 406-16.

18. Lawrie SM, Hall J, McIntosh AM, Owens DG, Johnstone EC. The 'continum of psychosis': scientifically unproven and clinically impractical. Br J Psychiatry 2010 ; 197(6) : 423-5.

19. Shoesmith WD. Correspondence. The classification of psychosis. Br J Psychiatry $2011 ; 198,323$.

20. Farias M, Underwood R, Claridge G. Unusual but sound minds: mental health indicators in spiritual individuals. Br J Psychology 2013 ; 104(3) : 364-81. 
21. Evrard R. Psychopathologie et expériences exceptionnelles: une revue de la littérature. L'Evolution Psychiatrique 2013a ; 78(1) : 155-76.

22. Claridge GS. Schizotypy: Implications for illness and health. New York : Oxford University Press ; 1997.

23. Schofield K, Claridge G. Paranormal experiences and mental health: Schizotypy as an underlying factor. Pers Ind Diff 2007 ; 43(7) : 1908-16.

24. Goulding A. Schizotypy models in relation to subjective health and paranormal beliefs and experiences. Pers Ind Diff 2004 ; 37(1) : 157-67.

25. Goulding A. Healthy schizotypy in a population of paranormal believers and experients. Pers Ind Diff $2005 ; 38$ : 1069-83.

26. McCreery C, Claridge G. Healthy schizotypy: the case of out-of-the-body experiences. Pers Ind Diff $2002 ; 32(1): 141-54$.

27. Allen MS. Beyond the happy schizotype: Opportunities for personal transformation in putatively pathogenic schizotypal experiences. Thèse de psychologie non publiée, Université de Miami, Etats-Unis ; 2008.

28. Nettle D. Schizotypy and mental health amongst poets, visual artist, and mathematicians. J Res Personality 2006 ; $40: 876-90$.

29. Chmielewski M, Bagby RM, Markon K, Ring AJ, Ryder AG. Openness to Experience, Intellect, Schizotypal Personality Disorder, and Psychoticism: Resolving the Controversy. J Pers Disorders 2014 ; 28(4) : 483-499.

30. Bradbury DA, Stirling J, Cavill J, Parker A. Psychosis-like experiences in the general population: An exploratory factor analysis. Pers Ind Diff 2009 ; 46(7) : 729-34.

31. Hori H, Teraishi T, Sasayama D, Matsuo J, Kinoshita Y, Otta M, Hattori K, Kunugi H. A latent profil analysis of schizotypy, temperament, and character in a nonclinical population: association with neurocognition. J Psychiatric Res 2014 ; 48(1) : 56-64.

32. Kernberg O. La thérapie psychodynamique des personnalités limites. Paris : Presses Universitaires de France ; 1995.

33. Kernberg O. Les troubles limites de la personnalité. Paris : Dunod ; 1997.

34. Kernberg O. Les troubles graves de la personnalité. Paris : PUF ; 2004.

35. Bienvenuto S, Siniscalo R. Entretien avec Otto Kernberg. Psythere 2004. Consulté le 07/07/14 sur : http://psythere.free.fr/article.php?id_article=33 (Orig. : Bienvenuto S, Siniscalo R. Psychoanalysis in America. Otto Kernberg. Journal of European Psychoanalysis $1997 ; 5)$.

36. Cattell RB, Eber HW, Tatsuoka MM. Handbook for the Sixteen Personality Factor Questionnaire (16PF). Champaign, IL: Institute for Personality and Ability Testing ; 1970.

37. Ahmed AO, Green BA, Goodrum NM, Doane NJ, Birgenheir D, Buckley PF. Does a latent class underlie schizotypal personality disorder? Implications for schizophrenia. J Abnormal Psychol 2013 ; 122(2) : 475-91.

38. Esterberg M, Compton M. The psychosis continuum and categorical versus dimensional diagnostic approaches. Current Psychiatry Reports 2009 ; 11(3) : 179-84.

39. Larkin W, Read J. Childhood trauma and psychosis: Evidence, pathways, and implications. J Postgraduate Medicine 2008 ; 54(4) : 287-93.

40. Read J, van Os J, Morrison AP, Ross CA. Childhood trauma, psychosis and schizophrenia: a literature review with theoretical and clinical implications. Acta Psychiatr Scandinavia $2005 ; 112: 330-50$.

41. Kocsis-Bogár K, Miklósi M, Forintos DP. Impact of adverse life events on individuals with low and high schizotypy in a nonpatient sample. J Nerv Mental Diseases 2013 ; 201(3) : 208-15. 
42. Evrard R. Schizotypie ou névrose ? la place des expériences réputées psychotiques. Perspectives psychiatriques 2010 ; 49(3) : 230-40.

43. Evrard R. Pour introduire la névrose extraordinaire. Recherches en psychanalyse, 2013b ; n 15 : 71-9. / Evrard R. Extraordinary Neurosis: An Introduction. Researches in Psychoanalysis $2013 \mathrm{~b} ; \mathrm{n}^{\circ} 15: 78 \mathrm{a}-86 \mathrm{a}$.

44. Arce-Ross G. Déclenchement des psychoses: les facteurs blancs, une valeur de nuisance vide. L'information psychiatrique 2003 ; 79(5) : 403-14.

45. Chaperot C. Structuralisme, clinique structurale, diagnostic différentiel névrosepsychose. Paris : L'Harmattan ; 2003.

46. Fellahian C. La psychose selon Lacan. Evolution d'un concept. Paris : L'Harmattan ; 2005.

47. Brett CMC, Peters EP, Johns LC, Tabraham P, Valmaggia LR, McGuire PK. Appraisals of Anomalous Experiences Interview (AANEX): a multidimensional measure of psychological responses to anomalies associated with psychosis. The $\mathrm{Br} \mathrm{J}$. Psychiatry $2007 ; 191(51): 23-30$.

48. Lacan J. Le Séminaire livre XXIII : Le sinthome. Paris : Seuil ; 2005.

49. Romme M, Escher S. Making sense of voices. London : Mind Publications ; 2000.

50. Guelfi JD. Axe 1, Axe 2 ou troubles mentaux et troubles de la personnalité. Evol Psychiatr $2014 ; 79: 55-67$.

51. Bourgeois ML. Avis de tempête su le DSM 5 (La nouvelle antipsychiatrie). L'Encéphale $2014 ; 40: 1-2$.

52. Tsuang MT, van Os J, Tandon R, Barch DM, Bustillo J, Gaebel W, Gur RE, Heckers S, Malaspina D, Owen MJ, Schultz S, Carpenter W. Attenuated psychosis syndrome in DSM-5. Schizophr Res $2013 ; 150(1): 31-5$.

53. Teyssier JR. Prodromes de la schizophrénie : consensus ou confusion? L'Encéphale 2013 ; 39(Suppl. 1.) : S1-S7.

54. Evrard R, Rabeyron T. Risquer la psychose : objections faites au «syndrome de psychose atténuée ». Psychiatrie, Sciences Humaines et Neurosciences 2012 ; 10(2) : 45-67.

55. Evrard R, Rabeyron T. Psychose pour tous : la jeunesse au risque du «syndrome de psychose atténuée »? Psychiatrie de l'Enfant $2014 ; 57(1): 331-48$.

56. Maleval JC. Limites et dangers des DSM. Evol Psychiatr 2003 ; 68 : 39-61. 